\title{
Meditation increases the depth of information processing and improves the allocation of attention in space
}

\author{
Sara van Leeuwen ${ }^{1}{ }^{*}$, Wolf Singer ${ }^{1,2}$ and Lucia Melloni ${ }^{1,2}$ * \\ 1 Department of Neurophysiology, Max Planck Institute for Brain Research, Frankfurt am Main, Germany \\ ${ }^{2}$ Ernst Strüngmann Institute (ESI) for Neuroscience in Cooperation with Max Planck Society, Frankfurt am Main, Germany
}

\section{Edited by: \\ Amishi P. Jha, University of Miami, USA}

\section{Reviewed by:}

Juliana Yordanova, Bulgarian Academy of Sciences, Bulgaria

Marieke Karlijn Van Vugt, University of Groningen, Netherlands

\section{*Correspondence:}

Sara van Leeuwen and Lucia Melloni, Department of Neurophysiology, Max Planck Institute for Brain Research, Deutschordenstraße 46, 60528 Frankfurt am Main, Germany. e-mail:vanleeuwen.sara@gmail.com; lucia.melloni@brain.mpg.de
During meditation, practitioners are required to center their attention on a specific object for extended periods of time. When their thoughts get diverted, they learn to quickly disengage from the distracter. We hypothesized that learning to respond to the dual demand of engaging attention on specific objects and disengaging quickly from distracters enhances the efficiency by which meditation practitioners can allocate attention. We tested this hypothesis in a global-to-local task while measuring electroencephalographic activity from a group of eight highly trained Buddhist monks and nuns and a group of eight age and education matched controls with no previous meditation experience. Specifically, we investigated the effect of attentional training on the global precedence effect, i.e., faster detection of targets on a global than on a local level. We expected to find a reduced global precedence effect in meditation practitioners but not in controls, reflecting that meditators can more quickly disengage their attention from the dominant global level. Analysis of reaction times confirmed this prediction. To investigate the underlying changes in brain activity and their time course, we analyzed event-related potentials. Meditators showed an enhanced ability to select the respective target level, as reflected by enhanced processing of target level information. In contrast with control group, which showed a local target selection effect only in the P1 and a global target selection effect in the P3 component, meditators showed effects of local information processing in the P1, N2, and P3 and of global processing for the N1, N2, and P3. Thus, meditators seem to display enhanced depth of processing. In addition, meditation altered the uptake of information such that meditators selected target level information earlier in the processing sequence than controls. In a longitudinal experiment, we could replicate the behavioral effects, suggesting that meditation modulates attention already after a 4-day meditation retreat. Together, these results suggest that practicing meditation enhances the speed with which attention can be allocated and relocated, thus increasing the depth of information processing and reducing response latency.

Keywords: meditation, hierarchical processing, EEG, ERP, source localization

\section{INTRODUCTION}

Attentional resources are limited and constrain the capacity to process information. In particular, there is a limit to how quickly attention can be allocated and reallocated to a different object or parts of the same object (Hopf et al., 2006). This limitation becomes particularly evident when considering that the visual world is intrinsically organized in a hierarchical manner. For instance, a forest has trees, and a tree in turn is composed of leaves. This example reflects the ubiquitous embedded relation between global and local parts present in the world. When attending to the global shape of an object, such as a tree, there is less attention available to attend to the fine grained detail, such as the leaves, and redirecting attention between levels - from the global shape to the local details or vice versa - is known to be inherently slow. In psychophysical tests subjects are typically much faster in detecting the global pattern than the local detail; this phenomenon known as the "global precedence effect" (Navon, 1977) clearly illustrates the limitations in the speed of allocation of attention between the local and the global level.
For long, such limitations in attention were thought to be unmodifiable (Kastner and Ungerleider, 2000; Chun and Marois, 2002). However, recent work has challenged this notion by showing that attention can indeed be trained. For instance, when subjects are trained to detect the local detail of an image, they can learn to detect it as quickly as the global shape, overcoming the global precedence effect (Dulaney and Marks, 2007). However, these types of training are specific to the stimulus material or the task that was practiced, showing little or at best limited transfer to new tasks, considerably restricting the benefit of training (Chun, 2000; Dulaney and Marks, 2007; Makovski et al., 2008; Kelley and Yantis, 2009). Notably, more flexible or integrated training regimes that involve constant switching of processing priorities, stimulus material, and regular adjustment to diverse and new task demands might lead to greater transfer across contexts (Bherer et al., 2005). In line with this proposal, it has been shown that real world training paradigms such as action video game playing are associated with more general training effects (Rauscher et al., 1993; Green and Bavelier, 2003, 2008; Jancke, 2009). Those improvements are 
seen across several tasks and cognitive functions like visuo-spatial capacity, visual acuity, visual attention, task switching, decision making, speed of processing, etc. (Bavelier et al., 2011). However, improvements in task performance in video game players have also been associated with undesirable outcomes, such as increments in aggressive behavior (Lemmens et al., 2011) and the risk of addiction (Weinstein, 2010). Meditation might be a valuable alternative as its practice involves not only training attentional (Lutz et al., 2008b) but also emotional regulation (Lutz et al., 2008a), thus promoting emotional balance and well-being.

During meditation attention is engaged onto an object for extended periods of time. When distraction occurs the practitioner is trained not to cling to the object of distraction but rather to disengage attention from it and to reengage attention onto the object of focus (Jha et al., 2007; Lutz et al., 2008b). Therefore, meditation is hypothesized to train the ability to engage and disengage attention from target stimuli in response to task demands (Lutz et al., 2008b). In line with this idea, recent studies have shown that the practice of meditation exerts rather general effects on attention (Jha et al., 2007; Lutz et al., 2008b; Slagter et al., 2011). For example, meditation improves the allocation of attention in the temporal domain, as measured by the attentional blink task (Slagter et al., 2007; van Leeuwen et al., 2009), and the capacity to sustain attention for prolonged periods of time (Carter et al., 2005). Evidence thus suggests that meditation might be a way to overcome capacity limitations of attention in a general manner, while also promoting well-being and positive emotions. Furthermore, meditation does not impose high demands on the practitioner, e.g., high dexterity or visual acuity as in video game playing, making it a suitable practice across the lifespan.

Here, we test the prediction that meditation may exert general and enduring modifications on attention by virtue of training the ability to allocate and reallocate attention. We evaluated this prediction in a global-to-local task, in which subjects must attend to either the global pattern or the local elements of a stimulus in order to successfully detect a target item. Specifically, we investigated the effects of two commonly practiced meditation styles: focused attention (FA) meditation and open monitoring (OM) meditation (Lutz et al., 2008b). During FA meditation practitioners develop the ability to attend to a relatively narrow range of items as attention is sustained on a chosen object, such as the breath. Once the ability to sustain attention on an object is mastered, the ability to monitor attention and to protect it from distraction becomes the main point of transition into OM meditation. Here, the focus is released and the practitioner becomes attentive moment-bymoment to anything that occurs in experience (Lutz et al., 2008b). Considering this fundamental difference in meditation style and in the aperture of the focus of attention, we expected that increasing the aperture of visual attention during OM meditation would facilitate global processing while decreasing the aperture during FA meditation would facilitate local processing (Stoffer, 1994; Lin et al., 2008). To investigate this, we tested two groups of meditation practitioners. We expected a group of highly trained monks and nuns with experience in both practices to show a reduction in global precedence, as they are equally trained on FA and OM meditation, while we expected to find a bias toward local stimulus processing in less experienced meditators with training in FA meditation only. In a longitudinal design, we exposed the latter group to a 4-day OM meditation retreat, predicting that, as the practitioner is trained to release the narrow focus of attention established during FA meditation, the local processing bias should wash out as the aperture of attention increases.

In addition, we measured event-related potentials (ERPs) in a group of highly trained Buddhist monks and nuns to investigate the underlying changes in brain activity and their time course related to the effects of meditation on hierarchical stimulus processing and attentional selection in the global-to-local task. Meditation has been shown to affect bottom-up attention and early sensory processing of information by improving perceptual discrimination and reducing inter-sensory facilitation effects (MacLean et al., 2010; van den Hurk et al., 2010b). Therefore we expected to see changes already at the level of the early components of the visual evoked response, such as the P1 and N1. For example, as the $\mathrm{P} 1$ has previously been found to be selective for local target information (Evans et al., 2000), we expected changes in bottom-up processing to be reflected in differences in the magnitude, latency, or laterality of this component between the two groups. In addition, previous work has shown that meditation can improve the ability to rapidly and efficiently allocate attention in time (Slagter et al., 2007; van Leeuwen et al., 2009), such that meditators exhibit a much diminished and sometimes even absent attentional blink. In these studies, analysis of the ERP time course revealed that meditators were better able to distribute attentional resources between the first and second target, suggesting a faster reallocation of attention in time (Slagter et al., 2007). We thus expected that, by virtue of training the mechanisms of attentional engagement and disengagement, meditators would display an enhanced ability to engage attention onto the target level (global/local) of a compound stimulus and reallocate it to the respective other level, effectively reducing the global precedence effect. We expected that this enhanced ability would correspond to a more frequent selection of target level information in the meditators, as measured in the ERP.

The results confirm that the practice of meditation strongly affects the allocation of attentional resources in space, resulting in a significant reduction, or even complete reversal, of global precedence, depending on the specific meditation style that is practiced. This behavioral advantage was mirrored electrophysiologically, (at the ERP level) by earlier and more frequent selection of target level information in the meditator group, which together suggest that meditation alters both the uptake of information at the level of early visual processing and the depth of processing.

\section{MATERIALS AND METHODS PARTICIPANTS}

Eight Buddhist monks and nuns with, on average, 5 years (SD 2 years) training in both FA and OM meditation practices were recruited from the Vietnamese Zen center in Frankfurt am Main, Germany (mean age 29 years; SD 9; 3 female). In addition, eight age and education matched control participants with no meditation experience (mean age 29 years; SD 8; 3 female) participated in the cross-sectional EEG study. For the longitudinal study, six meditation practitioners with, on average, 3 years (SD 1 year) experience in FA meditation practices were recruited from the Vietnamese 
Zen center in Frankfurt am Main, Germany (mean age 50 years; SD 10; 4 female). These participants were recruited from a group of meditators who take part in a weekly FA meditation course and had regular practice in FA meditation. The control group that also took part in the longitudinal study consisted of six age and education matched participants with no previous meditation experience (mean age 50 years; SD 9; 4 female). All participants were righthanded, had normal or corrected-to-normal visual acuity and informed consent was obtained from each participant before the experiment.

\section{STIMULI AND PROCEDURE}

E-Prime software (version 1.0.2) was used for stimulus presentation and response collection. Compound stimuli were used which consisted of a large number made up of small numbers. Numbers $1,2,3$, and 4 were used at both levels in eight different stimulus configurations shown in Figure 1A. Numbers 1 and 2 served as the target number, which could appear at the global or local level with equal probability. Numbers 3 and 4 served as neutral distracters and appeared at the global level if the target numbers appeared at the local level and vice versa. Stimuli were displayed in black at the center of the screen on a grey background. The global numbers subtended approximately $5.3^{\circ} \times 1.3^{\circ}-2.3^{\circ}$ of visual angle in the vertical and horizontal planes, respectively. The local stimuli occupied approximately $0.38^{\circ}$ of visual angle. Subjects were seated $70 \mathrm{~cm}$ from a computer monitor in a darkened room.

Figure 1B illustrates the experimental paradigm. Each trial began with the presentation of a central fixation cross that remained on the screen for a random duration between 1 and $1.5 \mathrm{~s}$. This was followed by the presentation of the compound stimulus ( $100 \mathrm{~ms})$ after which a blank was presented until subjects responded. Subjects had to indicate as quickly and accurately as possible whether the target number ( 1 or 2 ) appeared at the global or local level via button press on a standard keyboard. When subjects saw the target number 1 or 2 they pressed the response key that corresponded to that target number, independent of the level at which the target appeared. The experiment was divided into 8 blocks of 128 trials. In total 1024 trials were collected. Subjects were allowed to pause between blocks. The experiment lasted approximately $45 \mathrm{~min}$. Before the experiment, subjects practiced the task in 24 trials.

In the longitudinal study both the group of meditation practitioners as well as the group of controls participated twice with a 4-day interval. Only the meditation practitioners took part in an OM meditation retreat during the 4-day interval.

\section{ELECTROPHYSIOLOGICAL RECORDINGS}

For the cross-sectional study, EEG was recorded in an electrically shielded, sound attenuated, dark cabin. EEG was recorded using a 129-channel Geodesic Sensor Net and amplified with an AC-coupled high input impedance (200 M $\Omega$ ) amplifier (Geodesic EEG System 200; Electrical Geodesics Inc., Eugene, OR, USA). Data were acquired with NetStation ${ }^{\mathrm{TM}}$ software. EEG was digitized at $1000 \mathrm{~Hz}$, and stored on a hard drive. The scalp impedance of each sensor was kept below $50 \mathrm{k} \Omega$, as suggested by the manufacturer. Horizontal and vertical eye movements were monitored by means of electrodes placed at the outer canthi of the eyes and the
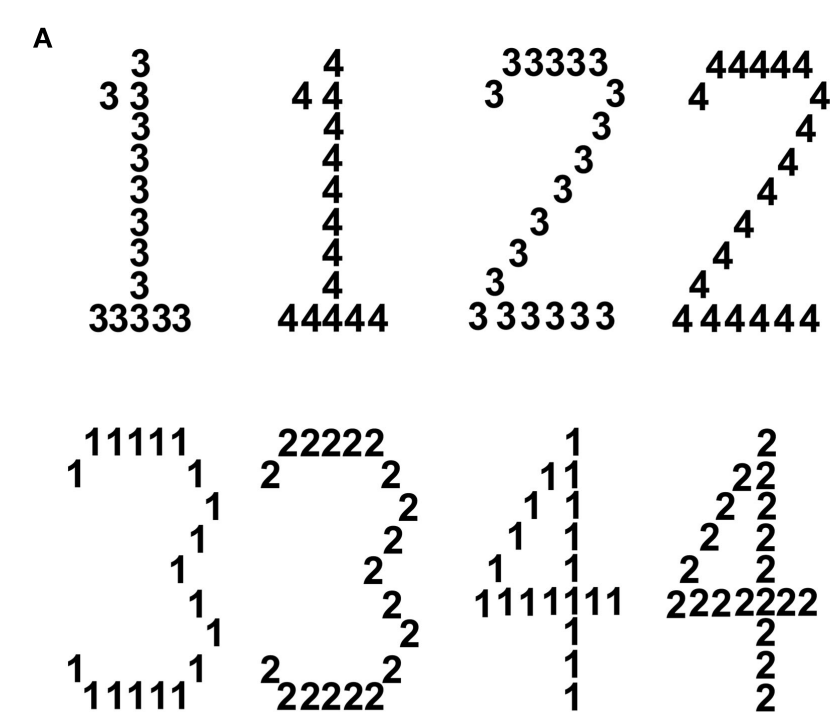

$\mathbf{B}$

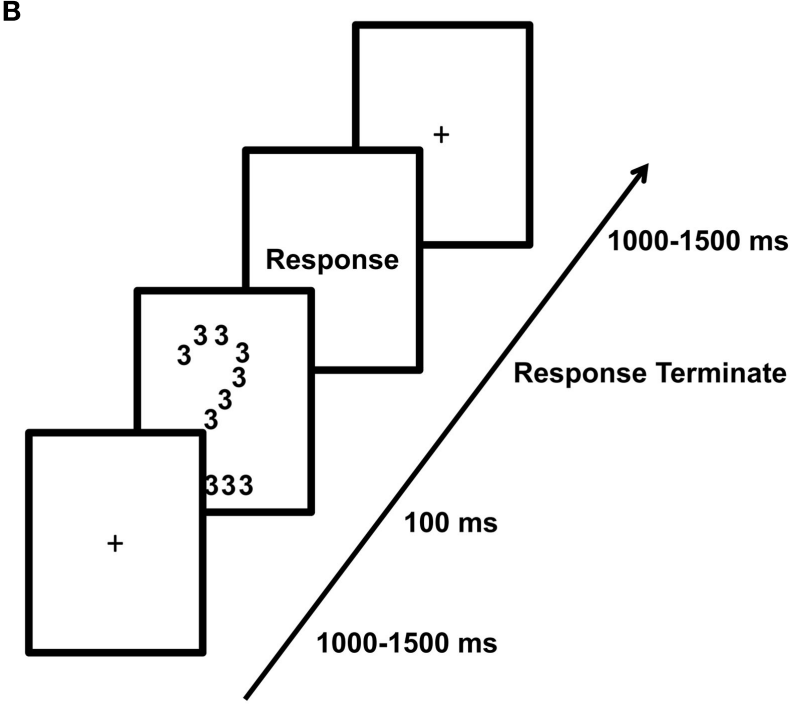

FIGURE 1 | Outline of the global-to-local paradigm and stimuli. A trial started with the presentation of a fixation cross lasting between 1 and $1.5 \mathrm{~s}$ followed by the presentation of one of eight possible stimuli displayed in (A). Subjects had to detect the target numbers 1 and 2 which appeared either at the global level or at the local level of the hierarchical stimulus and respond as quickly as possible via button press. They were instructed to press the " $Z$ " key when the target 1 appeared and the "M" key when the target 2 appeared. The stimulus itself was presented for $100 \mathrm{~ms}$ and replaced by a blank shown until the subject responded via a button press. (B) Outline of a single trial.

superior and inferior orbits. All electrodes were referenced online to the vertex.

\section{DATA ANALYSIS}

\section{Statistical analysis of the behavioral data}

Only correct responses were considered for analysis. Analysis of variance (ANOVA) was performed on averaged correct responses (RTs) between 200 and $2000 \mathrm{~ms}$. For the longitudinal 
and cross-sectional data analysis we ran a mixed ANOVA with the between-subject factor group (meditators, age matched controls) and within-subject factors precedence (global, local). The withinsubject factor session (before and after 4-day interval) was included in the analysis of the longitudinal data.

\section{EEG data analysis}

The continuous EEG was filtered with a bandpass $(0.3-30 \mathrm{~Hz})$ finite impulse response filter (Kaiser type). The EEG to compound stimuli was extracted in epochs of $1300 \mathrm{~ms}$ with a $500-\mathrm{ms}$ prestimulus baseline. Trials containing voltages exceeding $200 \mu \mathrm{V}$, or gradients exceeding $55 \mu \mathrm{V}$ were rejected, and bad channels were interpolated. Trials were visually inspected to reject cases with potential drifts, eye movements or electromyographic activity. Artifact free trials were averaged across the same stimulus category (local target/global target), baseline corrected over a 200ms window, and digitally transformed to an average reference. The EEGLAB MATLAB toolbox was used for visualization and topographic plots (Delorme and Makeig, 2004).

\section{Statistical analysis of ERPs}

We carried out two complementary analyses on the ERPs to gain a full understanding of the event-related data. First, we analyzed the mean amplitude of P1 (55-90 ms), N1 (130-190 ms), N2 (200-250 ms) and P3 (250-400 ms). We focused on those ERP components as previous studies have related them to global/local processing (Han and Chen, 1996; Proverbio et al., 1998; Evans et al., 2000; Conci et al., 2011) and we aimed at investigating whether meditators and controls differed in ERPs known to reflect hierarchical stimulus processing (global/local relations). However, one drawback of this approach is that differences that lie outside the investigated ERP components remain undetected. To circumvent this limitation, we ran a second, exploratory analysis. To investigate whether there were electrodes/time bins that were different from the potentials discussed before but were also sensitive to group differences in local/global processing; we conducted a whole-brain analysis. In this analysis, we systematically examined group differences in all regions that showed a significant difference between local and global processing. For both analyses we restricted our analysis window to 400-ms post stimulus to avoid confounds from motor response execution that occurred on average at 421 and $543 \mathrm{~ms}$ in the meditator and the control population, respectively.

To increase statistical power of the component-based analysis, mean amplitude was computed over groups of electrodes representative of the topography of each scalp component. Regions of interest (ROIs) were selected based on the compound waveform of all conditions to minimize selection bias. We identified an occipital P1 (electrodes: 58, 59, 65, 66, 70, 83, 84, 90, 91, 96) and N1 (58, $64,65,69,70,71,82,83,89,90,95,96)$, a lateral occipital N2 (64, $65,68,69,89,90,94,95)$ and a centroparietal P3 $(31,37,42,52,53$, 54, 55, 61, 62, 67, 77, 78, 79, 80, 86, 87, 129). Figures 3A, 4A, 5A and $6 \mathrm{~A}$ illustrate the topographies and selected electrodes for each component. As for the behavioral data, we analyzed the ERP with a mixed ANOVA with precedence (local, global) and hemisphere (left, right), when applicable, as within-subjects factors and group (meditators, control) as between-subject factor.
To evaluate effects not captured by the component-based ERP analysis we carried out a data-driven analysis, in which we first collapsed the data from the meditator and control group and then calculated a $t$-test at $p=0.01$ of precedence (Local vs. Global) per time point and electrode. We applied a cluster threshold that included only those electrodes and time points that were significant over a period of $15 \mathrm{~ms}$ or more over at least two contiguous electrodes (Thorpe et al., 1996; Dehaene et al., 2001; Murray et al., 2001). Anterior effects (frontal electrodes) were excluded due to the potential contamination by eye movements/blinks in these regions. Figure 7 illustrates the electrodes and topographies of each of the five clusters revealed with this procedure. Precedence effects were observed over temporal electrodes $(50,56,57$, 64) between 45 and $75 \mathrm{~ms}$, over frontal electrodes $(11,12,19)$ between 65 and $80 \mathrm{~ms}$, over right occipital electrodes (82, 83, 88, $89,90)$ between 140 and $190 \mathrm{~ms}$, over centroparietal electrodes $(31,52,53,54,59,60,61,62,66,70,71,72,77,78,79,80,86$, 87 ) between 255 and $290 \mathrm{~ms}$, and finally over centroparietal electrodes $(50,51,52,53,58,59,60,61,65,66,67,69,70,71,74$, $76,77,78,82,83,84,85,86,89,90,91,92,96,97)$ between 380 and $400 \mathrm{~ms}$. Mean amplitudes per cluster were then submitted to a repeated measures ANOVA with factors precedence and group.

Finally, to determine whether behavior covaried with ERP amplitude, we correlated the behavioral difference in response times between global and local targets with the difference in ERP amplitude responses to global and local targets for each time bin, as determined by the data-driven analysis (using Pearson's correlation).

\section{Source reconstruction analysis of the EEG data}

To identify the brain areas involved in processing precedence and their differential modulation by group we estimated source locations of the ERP signals using SPM 8 (http://www.fil.ion.ucl.ac.uk/spm/software/spm8/). We selected four time windows that included periods from the componentbased and data-driven ERP analysis: 45-90, 130-190, 200-250, and 250-400 ms. Three-dimensional source reconstruction of ERP signals were performed by the Multiple Sparse Priors (MSP) approach (Friston et al., 2008). A tessellated cortical mesh was created per subject using a template brain of the Montreal Neurological Institute (MNI). This mesh containing 8196 vertices served as a brain model to estimate current source distribution. Positions of current dipoles were restricted to the cortical surface and were evenly placed at each node of the mesh. This dipole mesh was spatially co-registered with the electrode locations. Then, we constructed a head spherical model for the forward solution for each data set after which an inverse reconstruction with the MSP approach was calculated for each time period of interest. The inverse reconstruction was performed for each experimental condition (global target condition, local target condition) and group (meditators, controls) separately via a group inversion step where the condition specific ERPs of all subjects in a group were inverted together to ensure consistency over the individual models. This resulted in four separate inverse reconstruction solutions for which sources were normalized and common to all participants within each group for each condition. Furthermore sources were computed 
over the whole frequency spectrum that our ERPs contained, namely $0.3-30 \mathrm{~Hz}$.

For the second-level (group level) analysis, the resultant MSP images were submitted to a random-effect analysis in SPM in a full factorial design with group as between-subject factor, and condition, as within-subject factor. An F-test was calculated per time period to investigate an interaction effect between group (meditators, controls) and condition (global target, local target), thresholded with family wise error (FWE) correction at $p<0.05$. $p$-Values for each cluster of source activity in the stimulus-locked analysis, and corresponding MNI coordinates, are displayed in Table 1. $t$-Test per group were followed to further investigate the direction of the interaction.

\section{RESULTS}

\section{BEHAVIORAL PERFORMANCE}

In the cross-sectional study, meditators tended to perform the task faster than controls, with an overall advantage of $\sim 122 \mathrm{~ms}$ $\left[F_{(1,14)}=4.28, p=0.057\right.$, meditators: $421 \mathrm{~ms}$, controls $\left.=543 \mathrm{~ms}\right]$. This advantage was not accompanied by decrements in performance, as accuracy was similarly high in both groups, speaking against a speed-accuracy trade off, and pointing to an increased speed of processing in the meditator group. We found a significant effect of global precedence on reaction times $\left[\mathrm{RT} ; F_{(1,14)}=23.47\right.$, $p<0.01]$ and on accuracy $\left[F_{(1,14)}=7.13, p<0.05\right]$. As expected, targets appearing at the global level were detected, on average, 40 ms faster (global: 462.4, local: 501.4) but also more accurately (global: 98\%, local: 96\%) than targets appearing a the local level, replicating the typical global precedence effect (Navon, 1977; Han and Chen, 1996; Proverbio et al., 1998; Evans et al., 2000). Importantly, the size of the precedence effect significantly differed between meditators and controls $\left[F_{(1,14)}=4.72, p<0.05\right]$. As can be seen from Figure 2A, the global precedence effect was more than halved in the meditators as compared to controls (global < local, meditators: $21.5 \mathrm{~ms}$, controls; $56.4 \mathrm{~ms}$ ).

In the longitudinal study, we observed a significant 3-way interaction [group $\times$ target level $\times$ session $\left(F_{(1,10)}=6.6, p<0.05\right)$, see Figure 2B]. Figure 2B shows RT for global and local targets separated per group and session. As in the cross-sectional study, the control group exhibited a typical global precedence effect, i.e., subjects detected global targets faster than local targets, and this effect did not change with sessions $[T(5)=0.4$, $p=0.7 ; 48.3$ and $50.6 \mathrm{~ms}$ for sessions 1 and 2, respectively]. In contrast, the meditators showed a significant modulation of the precedence effect as a function of the form of meditation practiced $[T(5)=4, p=0.01]$ : As predicted, meditators with extensive FA meditation experience detected local targets significantly faster than global targets before the retreat (local $<$ global $=38.5 \mathrm{~ms}$ ). However, after the 4 -day OM meditation retreat, which is thought to train the enlargement of the focus of attention, the same subjects showed a strong reduction of local precedence (local $<$ global $=11 \mathrm{~ms}$ ). No effect on accuracy was found in either group [controls $=(T(5)=0.9, p=0.41)]$, $[$ meditators $=(T(5)=0.17, p=0.87)]$.

Together, these data show that the practice of meditation strongly affects the allocation of attentional resources in space, resulting in a significant reduction, or even complete reversal,

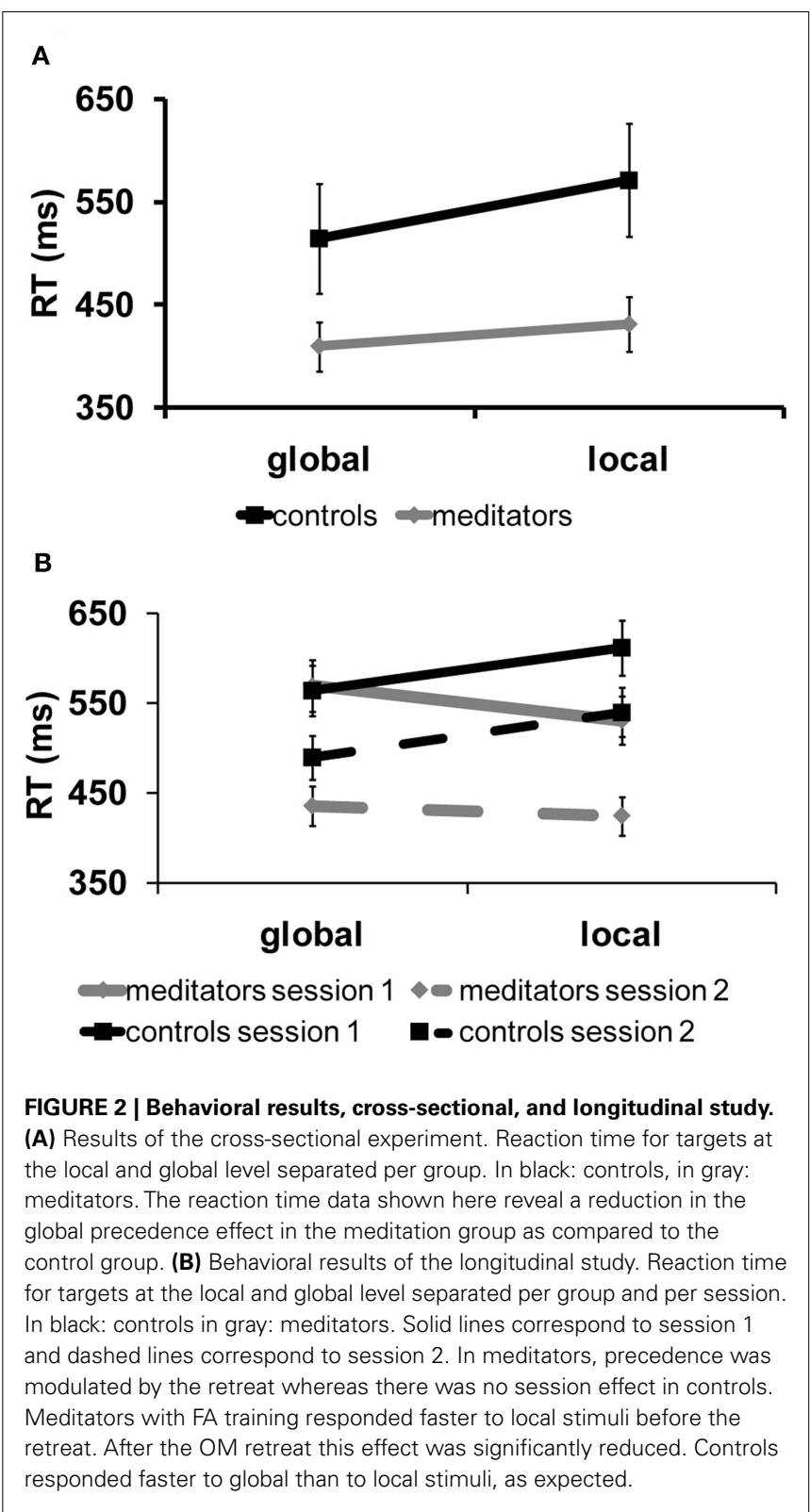

of global precedence depending on the specific meditation style that is practiced. Meditators were also generally faster than nonmeditators. As this occurs in the absence of any trade-off with accuracy, it suggests that meditation also affects the speed of processing. The results obtained in the longitudinal study are particularly reassuring as they rule out a common concern in meditation studies, i.e., selection bias.

\section{ERP EFFECTS}

To gain insight into how meditation affects the allocation of attentional resources in space and the speed of information processing, we compared ERP components between meditators and controls. In particular, we focused on the $\mathrm{P} 1, \mathrm{~N} 1, \mathrm{~N} 2$, and $\mathrm{P} 3$, which have previously been shown to be modulated by attentional selection in hierarchical processing (Han and Chen, 1996; Evans et al., 2000; 
Yamaguchi et al., 2000; Han et al., 2001; Volberg and Hubner, 2004).

P1 amplitude was higher for targets at the local level than for targets at the global level both in meditators and in controls $\left[F_{(1,14)}=6.75, p<0.05\right.$, local $=1.28 \mu \mathrm{V}>$ global $\left.=0.95 \mu \mathrm{V}\right]$. This result is in line with previous studies indicating attentional selection of local target level information already during the P1 component (Evans et al., 2000; Han et al., 2000; Jiang and Han, 2005). Importantly, however, this effect differed between groups and hemisphere $\left[F_{(1,14)}=4.77, p<0.05\right]$ (see Figures 3B,C). ANOVAs per group revealed that in the control group P1 amplitude was higher for local than for global targets only in the left hemisphere $\left[F_{(1,7)}=5.41, p=0.053\right.$, local $>$ global, left $0.52 \mu \mathrm{V}$ vs. global > local, right $0.1 \mu \mathrm{V}]$, supporting a left hemispheric predominance for local target processing found in previous studies (Fink et al., 1997). In contrast, in meditators P1 amplitude was higher for local than for global target in both hemispheres $\left[F_{(1,7)}=5.69, p<0.05\right.$; local $=1.9 \mu \mathrm{V}>$ global $\left.=1.5 \mu \mathrm{V}\right]$. Thus, in meditators local target information is selected at the level of the P1 in both the left and right hemispheres, while in controls selection was only found in the left hemisphere. This suggests that meditation already affects the earliest stages of information processing enabling simultaneous processing of both, local and global, target level information.

The amplitude of the $\mathrm{N} 1$ component was modulated by group, target level and hemisphere $\left[F_{(1,14)}=4.9, p<0.05\right]$. As can be seen in Figure 4, in the meditator group N1 amplitude was larger for global targets than for local targets in the right hemisphere, but

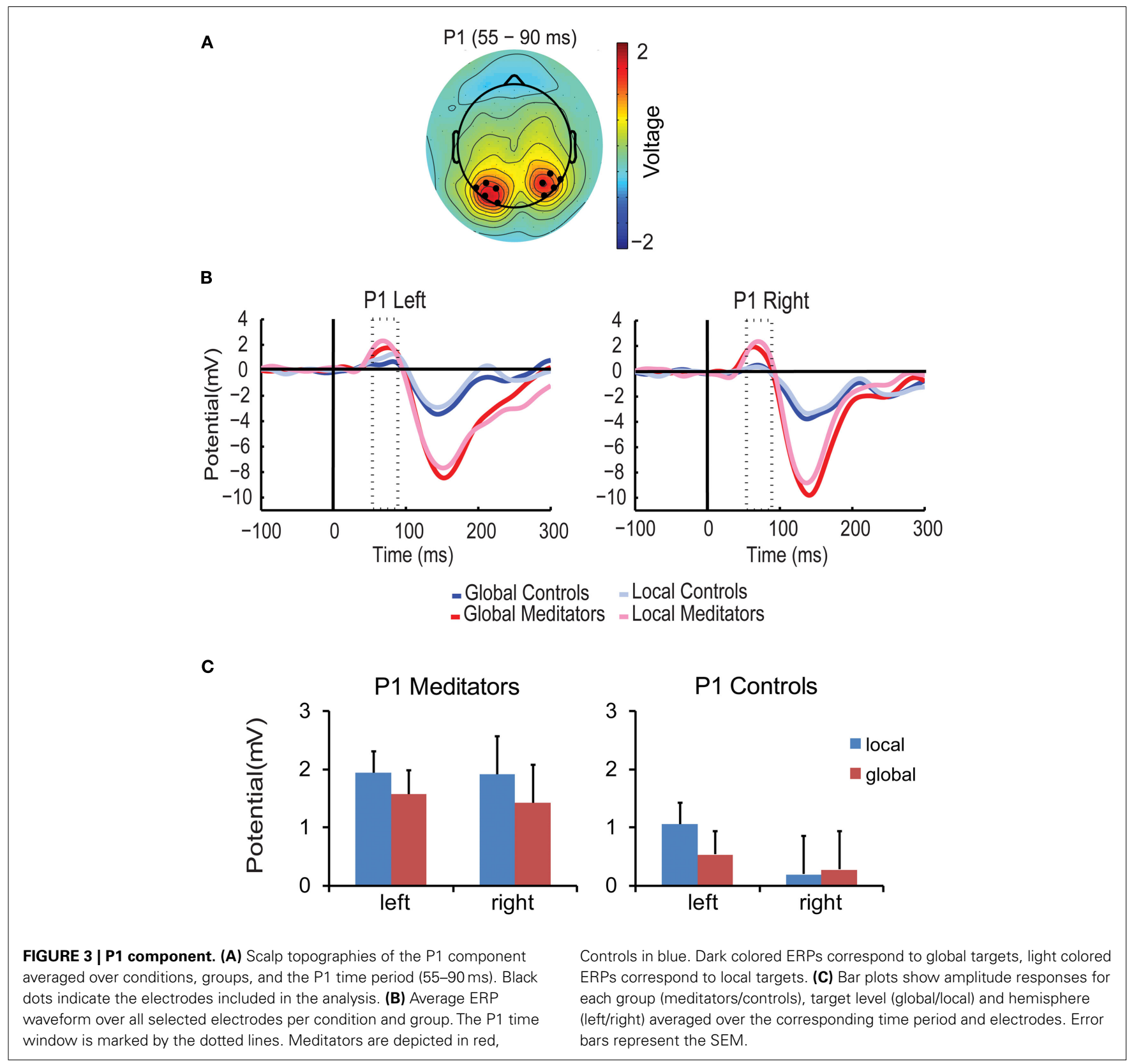




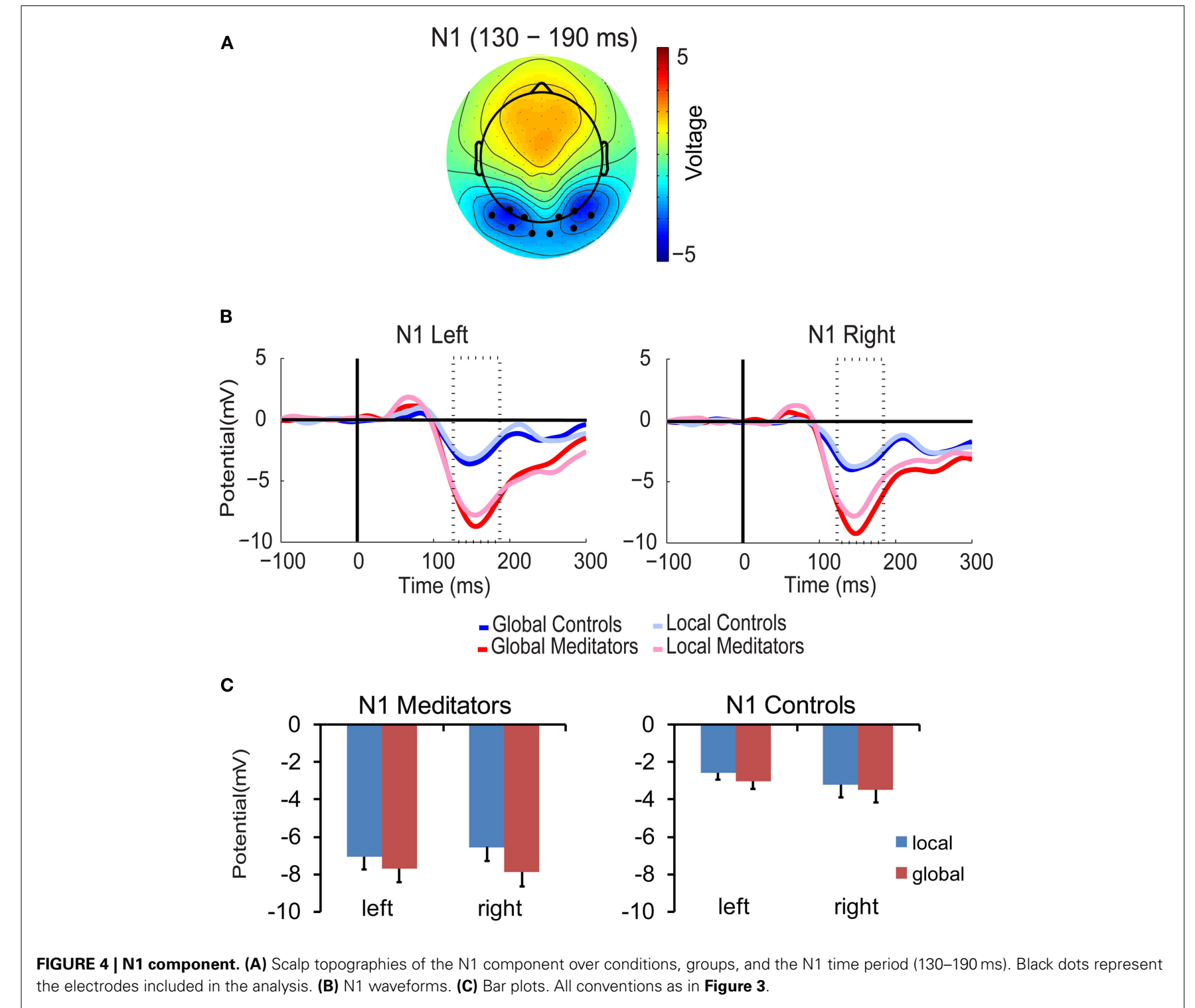

not in the left [target level $\times$ hemisphere $F_{(1,7)}=6.03, p<0.05$; global $>$ local, left $0.7 \mu \mathrm{V}$, right $1.4 \mu \mathrm{V}$ ]. Right hemispheric predominance for global targets has also been found in previous studies (Fink et al., 1997). In contrast, in the control group N1 amplitude showed no sign of hierarchical selection in both hemispheres (all $p>0.23$ ). Together, these findings show that meditators were already selecting global targets in the right hemisphere at around $150 \mathrm{~ms}$ while controls were not, thus suggesting that meditators began processing global target information earlier than controls.

The amplitude of the $\mathrm{N} 2$ component was also modulated by target level, hemisphere, and group $\left[F_{(1,14)}=7.03, p<0.05\right]$. As shown in Figure 5, in the meditators N2 amplitude was higher for global than local targets in the right hemisphere, while the opposite patterns was found in the left hemisphere, i.e., higher amplitude for local than global targets [target level $\times$ hemisphere $F_{(1,7)}=11.5, p<0.05$; local $>$ global, left $=0.2 \mu \mathrm{V}$; global $>$ local, right $=0.7 \mu \mathrm{V}]$. In contrast, in the control group no signs of hierarchical processing were found in either hemisphere (all $p>0.19)$. This finding suggests that only meditators were extracting global/local information during this time period.

P3 amplitude was higher for targets at the global level than for targets at the local in both groups $\left[F_{(1,14)}=6.33, p<0.05\right.$, mean global $=4.64 \mu \mathrm{V}$, mean local $=4.19 \mu \mathrm{V}$, see Figures $6 \mathrm{~B}, \mathrm{C}]$. This supports previous findings of global target selection in the P3 time period (Dalrymple et al., 2009; Machinskaia et al., 2010).

Thus, already at $400 \mathrm{~ms}$ meditators have processed hierarchical information (i.e., selection of local and/or global targets) earlier, more often, and have engaged more resources (as indicated by the absence of lateralization in the P1) than controls, which together suggest increased depth and speed of processing as a result of meditation practice.

\section{DATA-DRIVEN EFFECTS}

Although our experimental hypothesis focused on ERP components previously related to hierarchical processing, we conducted 


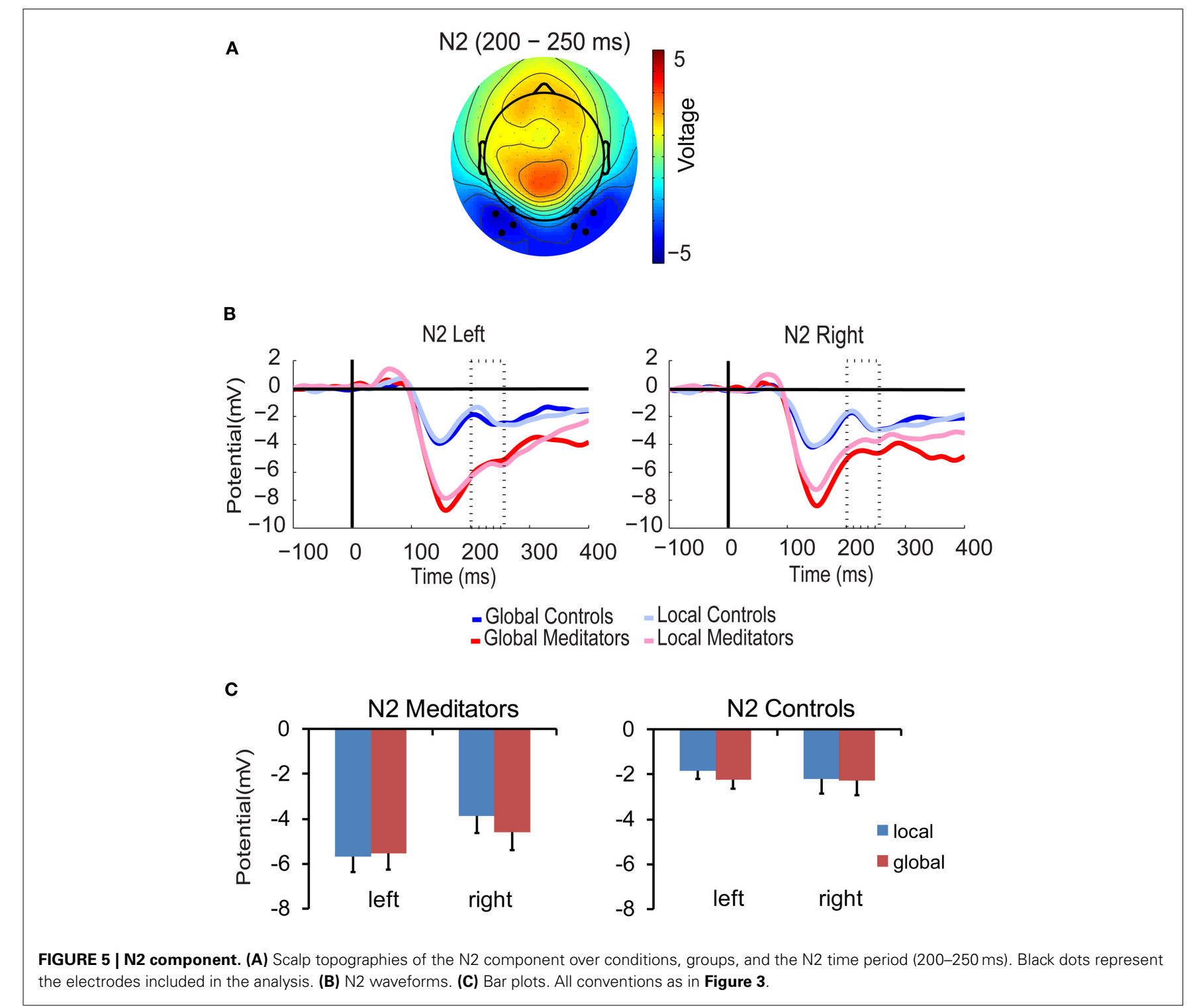

an exploratory analysis over the full montage of electrodes and time points because the pre-selection of electrodes, time periods, and components might have precluded important differences in hierarchical processing between groups. The exploratory analysis allowed us to map the sensors and time points during which responses exhibited target level related differences and then to compare the two groups for those regions and periods of interest. This procedure revealed five periods in which amplitudes differed between local and global targets: The earliest difference was observed at 45-75 ms over left temporo-occipital electrodes, with higher amplitudes for local than global targets $(0.355$ vs. $0.121 \mu \mathrm{V})$. Subsequently, higher amplitudes for local targets were also observed at $65-80 \mathrm{~ms}$ over frontal electrodes $(0.43$ vs. $0.037 \mu \mathrm{V})$. Later in the processing stream, response amplitudes were larger for global than for local targets over right occipital electrodes $(-5.1$ vs. $-4.2 \mu \mathrm{V})$ at $140-190 \mathrm{~ms}$ and also over centroparietal electrodes $(2.59$ vs. $1.59 \mu \mathrm{V})$ at $255-290 \mathrm{~ms}$. Finally, between 380 and $400 \mathrm{~ms}$ amplitudes were again larger for local than for global targets ( 2 vs. $1.19 \mu \mathrm{V})$ over centroparietal electrodes (see Figure 7). Thus, in line with the ERP components, higher amplitudes for local targets were observed earlier in the processing stream and predominantly over the left hemisphere. In contrast, global targets elicited higher amplitudes later on and were mostly right lateralized.

For all identified time periods and electrodes there was a significant interaction between group and target level [period $1\left(45-75 \mathrm{~ms}, F_{(1,14)}=9.79, p<0.01\right)$, period $2(65-80 \mathrm{~ms}$, $\left.F_{(1,14)}=16.24, p<0.001\right)$, period $3\left(140-190 \mathrm{~ms}, F_{(1,14)}=4.62\right.$, $p<0.05)$, period $4\left(255-290 \mathrm{~ms}, F_{(1,14)}=4.62, p<0.05\right)$, period $\left.5\left(380-400 \mathrm{~ms}, F_{(1,14)}=9.04, p<0.05\right)\right]$. Planned comparison revealed differential response amplitudes for global and local targets in periods $1-3$ and 5 for meditators that were not significant in controls (see Figure 7; period 1, 2, 3, 5 all $p<0.01$ in meditators and all $p>0.068$ in controls). In period 4 , i.e., between 
A

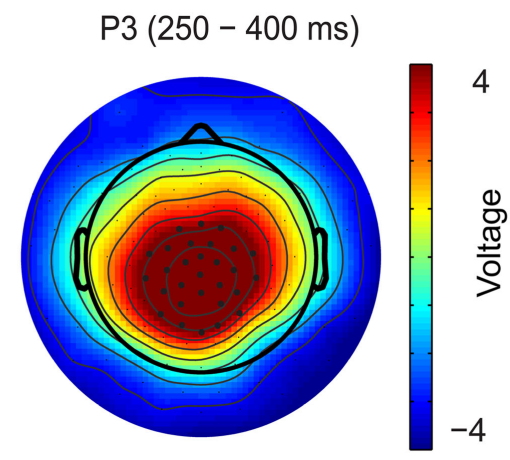

B

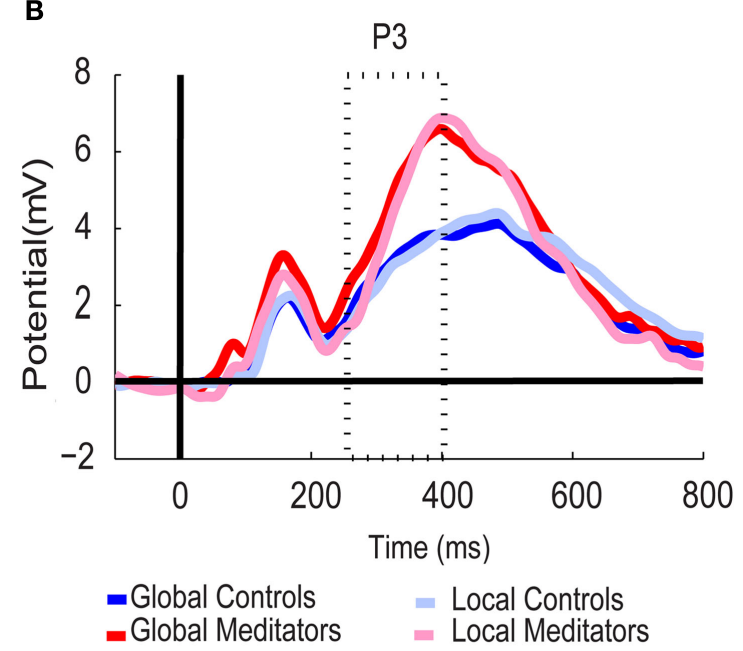

C

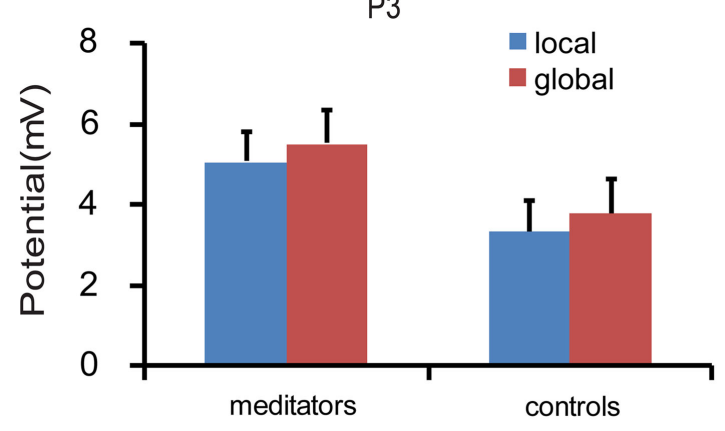

FIGURE 6 | P3 component. (A) Scalp topographies of the P3 component over conditions, groups, and the P3 time period (250-400 ms). Black dots represent the electrodes included in the analysis. (B) P3 waveforms. (C) Bar plots show amplitude responses for each group (meditators/controls) and target level (global/local) averaged over the corresponding time period and electrodes. Error bars represent the SEM.

255 and $290 \mathrm{~ms}$, both groups revealed larger response amplitudes for global than for local targets [meditators: $T(7)=4.44, p<0.01$; controls: $T(7)=2.3, p=0.054]$; however, this difference was more pronounced in meditators than in controls (see Figure 7D).

The topography and latency of the effect found between 45 and $75 \mathrm{~ms}$ coincides with an early time period within the $\mathrm{P} 1$, in which increased response amplitude to local targets was also observed
A

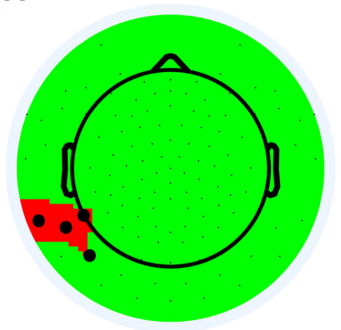

Precedence 45-75 ms

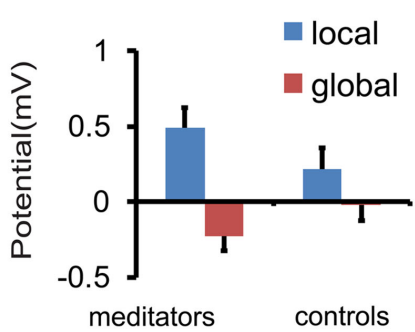

B

Precedence 65-80 ms
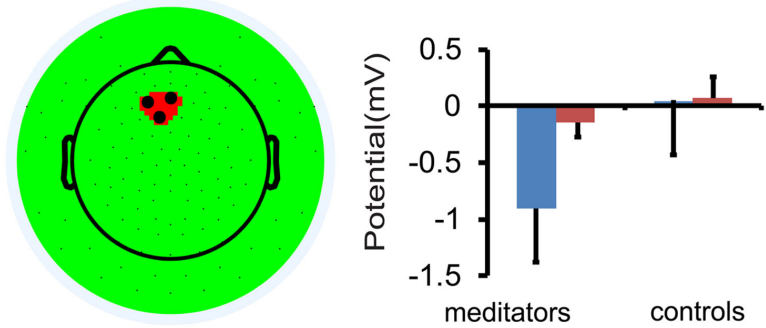

meditators

controls

C

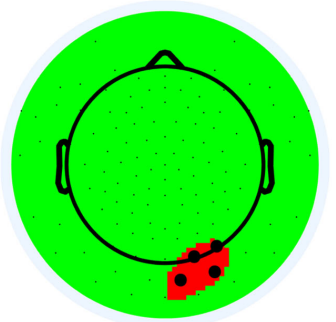

Precedence 140-190 ms

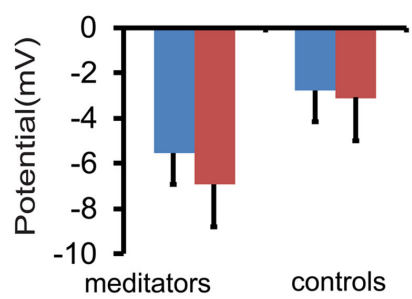

D

Precedence 255-290 ms
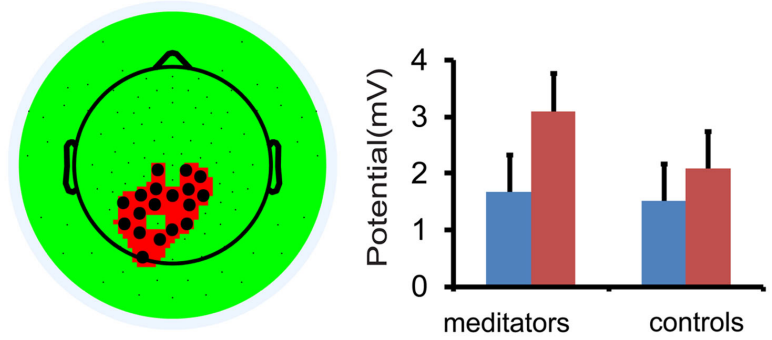

E

Precedence 380-400 ms
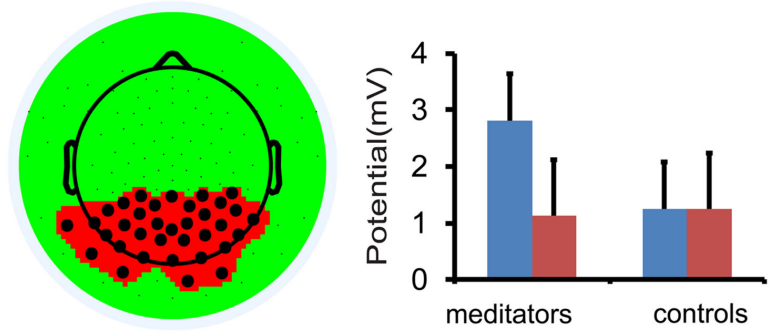

FIGURE 7 | Data-driven analysis based on precedence mapping. Each head plot shows the electrodes exhibiting an effect of precedence averaged over the corresponding time period. Each panel, (A-E), shows the amplitude difference between local and global targets (local-global) averaged over the corresponding time period and electrodes. Meditators are shown in red, controls in blue. Error bars represent the SEM. 
in both groups. Combined with the exploratory analysis, it suggests earlier selection of local targets in the meditator group as this effect was only observed in meditators in the early time window (45-75 ms). In addition, the topography and latency of the effect found between 140 and $190 \mathrm{~ms}$ coincides with the N1. Exactly like the N1, we found that meditators had increased response amplitude for global targets with a right lateralized response. In contrast to the early response to global target observed during the N1 time window in meditators, controls only begin to show global selection at a later time point, between 255 and $290 \mathrm{~ms}$. Together, these data indicates electrophysiological markers of local/global target selection with an earlier latency in meditators than in controls. In addition, evidence of local selection between 45 and $75 \mathrm{~ms}$, 65-80 ms, and 380-400 ms and global selection between 140 and $190 \mathrm{~ms}$ in the meditators but not in the controls suggests that while meditators are processing target level information at several different levels, controls are only processing global information once in the processing stream, at $255-290 \mathrm{~ms}$, a time period that overlaps with the P3.

\section{Correlation}

We found a significant correlation between the degree of global/local differences in the reaction times and the degree of global/local differences in ERP amplitudes at 60-85 ms over frontal electrodes $(R=-0.576, p=0.02)$ and again at 380-480 ms over centroparietal electrodes $(R=-0.644, p=0.007)$. Those subjects with a smaller "global precedence effect" in the reaction time data also had larger amplitude increases for local targets relative to global targets at $60-85$ and $380-480 \mathrm{~ms}$. This was not the case for any of the other time windows ( $p>0.05$; Figure 8).

\section{DISTRIBUTED SOURCE RECONSTRUCTION}

Taken together, the ERP components and the exploratory analysis point to earlier processing of target level information as well as increased depth of processing, i.e., more frequent target level selection and engagement of additional (neural) processing resources in meditators than in controls. To explore the neural sources underlying the improvement in performance in the global-tolocal task observed in meditators we performed Bayesian distributed source reconstruction analysis in four selected time periods (45-90, 130-190, 200-250, and 250-400 ms). These time periods subsume periods of the component based and the exploratory analysis. An interaction contrast between groups (meditators, controls) and target level (global, local) was performed for each period. This interaction contrast revealed brain areas for which target level effects were different between the two groups. To understand whether these differences were driven by higher activation in the meditation group or in the control group, we performed separate $t$-tests between the two target level conditions, per group. Reported results were all significant at $p<0.05$, FWE corrected for multiple comparisons. Table 1 contains the MNI coordinates for all sources identified in the interaction contrast, separated per period of interest. Given the uncertainty surrounding available reconstruction techniques, these results are exploratory and should be interpreted with caution. The results presented below should then be considered rough estimates of the underlying sources since our analysis did not consider individual differences in head shape or differences

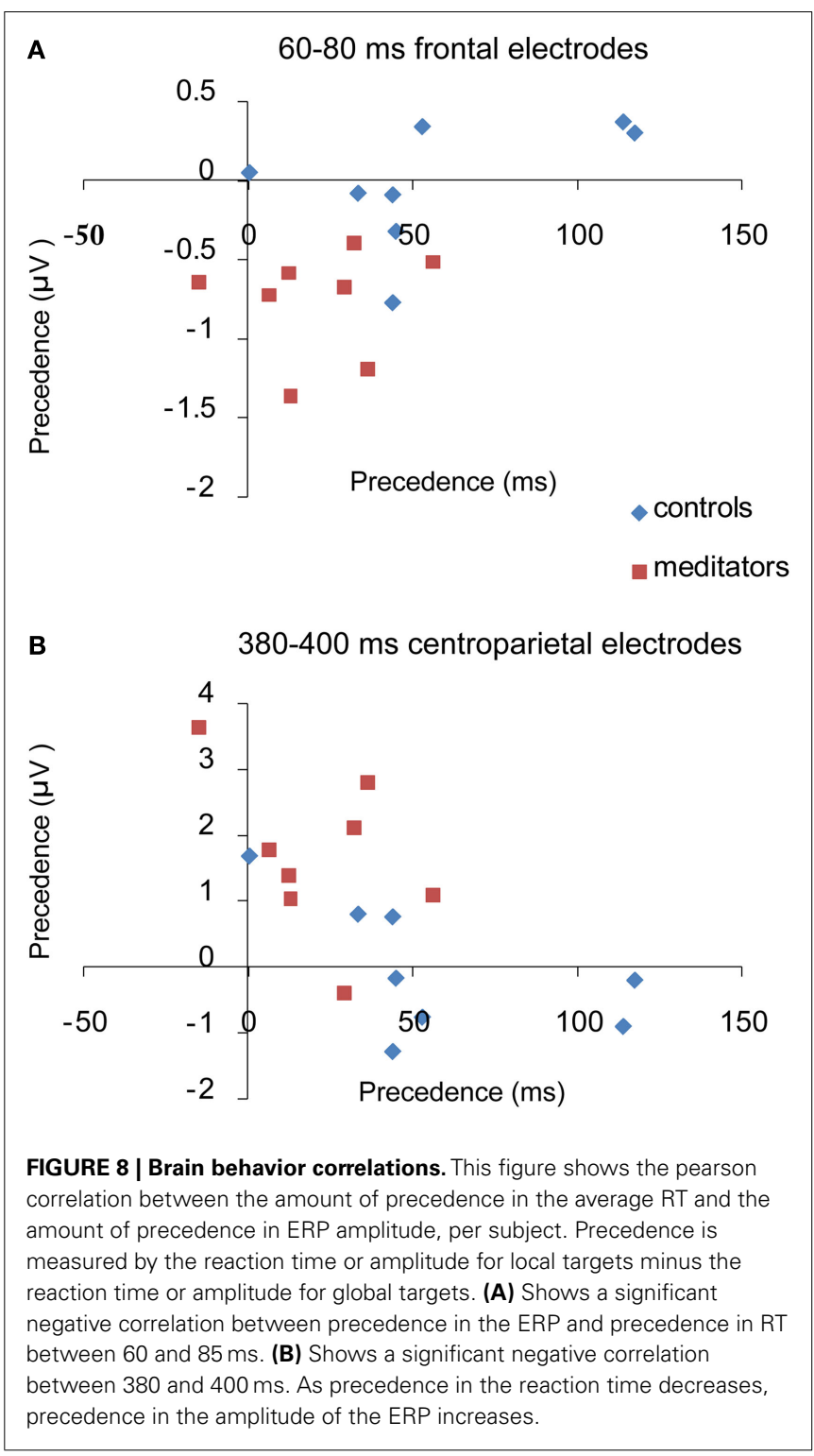

in brain anatomy, further limiting the spatial resolution of the source reconstruction.

Figure 9A shows the results of the interaction contrast for the time period between 45 and $90 \mathrm{~ms}$. Two significant clusters are observed: one in the left temporal parietal junction (TPJ) and another in the left dorsolateral prefrontal cortex (DLPFC). Both regions have previously been reported to be active during attention to global/local stimuli (Fink et al., 1997). Further $t$-tests showed that meditators were engaging the left TPJ more for local relative to global processing while controls were not (Figure A1A in Appendix). In the following time period (130-190 ms) we observed widespread differences across multiple cortical areas, spanning frontal, parietal, temporal, and occipital regions including the anterior cingulate cortex, precuneus and inferior occipital gyrus (Figure 9B) that have previously been found activated during other global-to-local tasks where the distractor is incongruent with the target (Weissman et al., 2002). $t$-Tests showed that these 

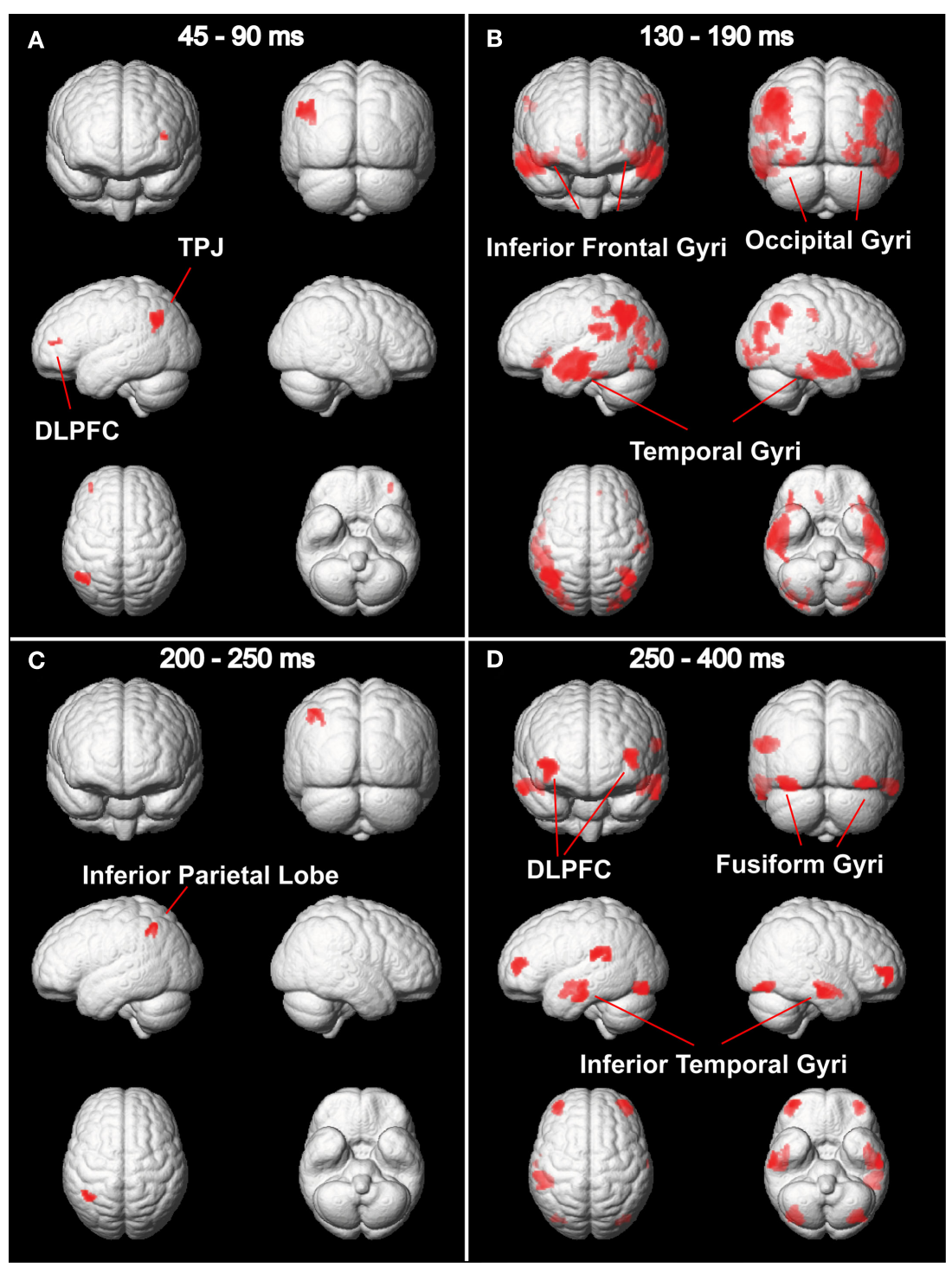

FIGURE 9 | Source reconstruction. Areas showing a significant group by target level effect, $p<0.05$, FWE-corrected. (A) Interaction effects were found between 45 and $90 \mathrm{~ms}$, corresponding to the latency of the P1, in the left TPJ and DLPFC; (B) between 130 and $190 \mathrm{ms,}$

regions were more active for global target processing in meditators but not in controls (Figure A1B in Appendix). Between 200 and $250 \mathrm{~ms}$ a significant cluster of differential activation was found in the left inferior parietal lobe (Figure 9C). $t$-Tests revealed that meditators displayed increased activation for global targets in the right and left middle temporal gyrus and predominantly in the left occipital regions for local targets. No effect was observed in controls (Figure A1C in Appendix). Finally, during the 250$400 \mathrm{~ms}$ window differences were primarily localized to bilateral clusters in temporal, occipital, and frontal regions, corresponding to the inferior temporal gyrus, inferior occipital/fusiform gyrus and DLPFC (Figure 9D), in line with previous studies (Fink et al., 1997; Weissman et al., 2002). Again, $t$-tests revealed differential and temporal gyri; (C) between 200 and $250 \mathrm{ms,} \mathrm{corresponding} \mathrm{to} \mathrm{the}$ latency of the N2, in the inferior parietal lobe and finally (D) between 250 and $400 \mathrm{~ms}$, corresponding to the latency of the P3, in the DLPFC, fusiform, and inferior temporal gyri. See Table 1 for $\mathrm{MNI}$ coordinates and number of voxels.

(global $>$ local) activation in the meditation group but not in the control group (Figure A1D in Appendix). This increase in activation for global target stimuli was found in inferior frontal regions. The overlap between the localization of activation patterns in the $t$-tests and the interaction contrast and the finding that target level differences could be found in the meditators but not in the controls suggests that the interaction effects found were indeed driven by increased target level effects in the meditation relative to the control population.

\section{DISCUSSION}

In summary, this study provides direct evidence that meditation alters spatial attention: while controls showed the typical global 
precedence effect, i.e., slower response times to local level targets as compared to global level targets (Navon, 1977), this effect was significantly reduced in the meditators. These results are in line with the hypothesis that meditation improves the allocation of attention in space and the ability to adjust the focus of attention from the larger, global pattern to the fine grained detail of an image. That is, meditation might alter the spatial distribution of attention by virtue of easing how fast the focus of attention is engaged and disengaged between spatial locations. Meditators did not only exhibit a strongly reduced global precedence effect, they were also overall much faster than controls, with an average advantage of more than $100 \mathrm{~ms}$. This effect cannot be explained by a speed-accuracy trade-off in the meditator population, as accuracy was overall very high and comparable between groups. Altogether, these results suggest increased speed of processing along with improvements in the distribution of attentional resources in the meditator population.

As expected, the two different types of meditation investigated here, FA and OM meditation, affected spatial attention differently. Meditators proficient in FA meditation, a practice expected to decrease the aperture of attention, showed faster responses to local target levels than to global target levels. However, upon completing a 4-day OM meditation retreat, the facilitation of local target selection washed out, concurring with the idea that OM meditation acts to expand the aperture of attention (Lutz et al., 2008b). Finally, meditators proficient in both FA and OM meditation revealed a reduction in global precedence and in overall reaction times, indicating that training both types of meditation facilitates local and global level processing and the ability to rapidly adjust the aperture of attention in response to task demands. Previous work supports the notion that different meditation styles affect attention differently (Valentine and Philip, 1999; Jha et al., 2007; Lutz et al., 2008b). For instance, it has been shown that while both FA and OM meditation enhance performance in a sustained attention task when stimuli are expected, only practitioners with experience in OM meditation show superior performance when stimuli are unexpected (Valentine and Philip, 1999). Altogether, this data suggests that FA and OM meditation have distinct effects on attention: while FA meditation trains more FA, OM meditation trains more distributed attention. For this reason, when investigating the effects of meditation on cognition, the homogeneity of the population under investigation is of crucial importance and the abilities trained during the specific practice must match the abilities necessary for the task.

To shed light on the functional changes underlying the improvements in performance in the meditator population we investigated the time course of electrophysiological signals related to hierarchical processing, combined with source localization to estimate the neural origin of those changes. The first signs of hierarchical processing were found during the time window of the P1 component. Concurring with previous studies, we found that controls and meditators exhibited higher P1 amplitudes in response to local targets than to global targets, suggesting that local information is already processed at the level of the P1 (Han and Chen,

Table 1 | Results of the full factorial source reconstruction analysis for each of the four time windows investigated.

\begin{tabular}{|c|c|c|c|c|c|c|c|}
\hline \multirow{2}{*}{$\frac{\text { Time period (ms) }}{45-90}$} & \multirow{2}{*}{$\begin{array}{l}\text { Side } \\
\text { Left }\end{array}$} & \multirow{2}{*}{$\begin{array}{l}\text { MNI coordinate region } \\
\text { Temporoparietal junction }\end{array}$} & \multicolumn{3}{|c|}{ Peak MNI coordinates } & \multirow{2}{*}{$\begin{array}{l}\text { No. of voxels } \\
230\end{array}$} & \multirow{2}{*}{$\frac{\text { P FEW-corrected }}{0.001}$} \\
\hline & & & -44 & -58 & 26 & & \\
\hline & Left & Dorsolateral prefrontal cortex & -36 & 44 & 12 & 30 & 0.018 \\
\hline & Right & Anterior temporal pole & 48 & 12 & -20 & 1188 & 0.000 \\
\hline & Right & Inferior parietal lobe & 36 & -56 & 40 & 542 & 0.000 \\
\hline & Right & Middle occipital gyrus & 28 & -90 & -4 & 137 & 0.000 \\
\hline & Left & Middle occipital gyrus & -52 & -74 & 0 & 67 & 0.004 \\
\hline & Left & Inferior frontal gyrus & -30 & 24 & -4 & 130 & 0.001 \\
\hline & Right & Inferior frontal gyrus & 46 & 18 & -10 & 147 & 0.009 \\
\hline & Right & Anterior cingulate & 8 & 32 & -10 & 68 & 0.000 \\
\hline & Left & Inferior temporal gyrus & -60 & -18 & -28 & 351 & 0.000 \\
\hline & Left & Superior temporal gyrus & -54 & -42 & 18 & 400 & 0.000 \\
\hline & Right & Dorsolateral prefrontal cortex & 42 & 54 & -12 & 285 & 0.002 \\
\hline & Left & Dorsolateral prefrontal cortex & -40 & 48 & 10 & 184 & 0.003 \\
\hline & Left & Fusiform gyrus & -42 & -76 & -18 & 259 & 0.006 \\
\hline & Right & Fusiform gyrus & 36 & -76 & -18 & 248 & 0.008 \\
\hline
\end{tabular}

Peak MNI coordinates for the interaction contrast between group (meditators vs. controls) and condition (global target vs. local target). Clusters surviving FWE-corrected threshold $p<0.05(F=30.2)$ and cluster size threshold $k=20$. 
1996; Evans et al., 2000; Han et al., 2000). Furthermore, neuroimaging and neuropsychological studies point to hemispheric asymmetries during hierarchical stimulus processing, with local target selection predominantly involving the left hemisphere, and global target selection primarily involving the right hemisphere (Robertson et al., 1991; Fink et al., 1997; Proverbio et al., 1998; Evans et al., 2000; Yamaguchi et al., 2000). This left hemispheric bias for local target selection has also been shown to occur as early as during the P1 (Evans et al., 2000). Our results confirm these previous findings by showing both increased $\mathrm{P} 1$ amplitude in response to local targets and a left hemispheric predominance in the control group. Importantly, the first differences between meditators and controls were already found in the P1 component. Meditators showed an earlier amplitude modulation, already at 45-75 ms. Additionally, the P1 enhancement to local targets occurred bilaterally and was not biased to the left. The bilateral P1 response is particularly interesting as it suggest on the one hand increased allocation of resources for the processing of local level information, but also the breakdown of the typical hemispheric asymmetry observed in controls. These findings point to an earlier uptake of local target information in meditators along with increased depth of local target information processing.

Similarly, previous work has shown that responses to global targets are enhanced during the time period of the P3 over centroparietal electrodes, suggesting that global target information is selected at the level of the P3 (Han et al., 2001; Dalrymple et al., 2009; Machinskaia et al., 2010). Our results replicate this finding in both the meditation and the control group. However, meditators already showed an effect of global target selection about $150 \mathrm{~ms}$ earlier during the N1 time period over occipito-temporal electrodes, which was absent in controls. Global target selection during the N1 time period has been previously reported, but mostly under conditions in which the to-be-attended level is known in advance (directed attention condition), e.g., when subjects are asked to detect a target exclusively at the global level while ignoring the local elements (Proverbio et al., 1998; Machinskaia et al., 2010). This earlier selection is thought to be related to top-down gain control mechanisms that boost global level information. As earlier processing of global shape was observed in meditators, this supports the notion of an earlier uptake of information in this population, this time for global targets, suggesting that meditation changed early sensory processing of both local (P1) and global targets (N1). Changes in sensory processing have previously been found in meditators, such as superior performance on a visual line discrimination task (MacLean et al., 2010). However, these changes have only been measured at the behavioral level. Here, we report evidence of functional changes in the brain associated with changes in the timing and magnitude of early visual components $(\mathrm{P} 1, \mathrm{~N} 1)$ in meditators with likely sources in the temporoparietal junction, inferior parietal lobe, inferior temporal gyrus, and inferior occipital gyrus.

In addition to the finding that meditators showed evidence of target level selection earlier in time, the current results further suggest that meditators were also able to process target level information at an increased depth as compared to controls. Here, we used the concept of depth of processing to refer to the increase in neural information processing which presumably reflects more elaborate processing at the functional level (Dehaene et al., 1998). This increase in information processing is evident from the finding that meditators showed more frequent target selection of both local and global target stimuli: While meditators showed enhanced ERP amplitudes in response to local targets in three different time periods during the processing stream $[\mathrm{P} 1, \mathrm{~N} 2$, and during the later part of the P3 (380-400 ms)], controls only showed evidence of local information selection at the level of the P1. In addition, meditators showed evidence of global target selection during the N1, N2, and P3, whereas controls only showed global target selection effects at the level of the P3. Thus, by $400-\mathrm{ms}$ post stimulus onset, meditators had shown local and global selection effects three times more often than controls. In face of the behavioral advantage observed in meditators, the earlier target level selection observed in the early visual components (P1, N1), the bilateral response at the level of the $\mathrm{P} 1$, the more frequent processing of target level information, the concurrent but hemispherically segregated processing of local and global information during the N2 time window, and finally the activation of higher order areas, e.g., frontal and parietal regions but also higher order sensory regions all point to an increased depth of processing and better allocation of attentional resources in the meditation population.

Our distributed source reconstruction further supports the hypothesis that meditation improves the ability to allocate attention in space. Neural sources of the electrical signals mostly localized to cortical networks that have previously been related to the allocation of attention during global/local processing (Fink et al., 1997; Yamaguchi et al., 2000; Weissman et al., 2002). This network comprises the TPJ, DLPFC and parietal regions that have previously been shown to be activated during attentional selection in hierarchical processing and have been linked to the top-down allocation of attention during the task (Fink et al., 1997; Yamaguchi et al., 2000; Weissman et al., 2002). The increased engagement of the temporoparietal junction is particularly interesting, as activity in this area has been found in hierarchical divided attention task where the level at which the target will appear is uncertain (Fink et al., 1997), as in our task. In particular, its activation has been related to attentional control processes which enhance neural responses in sensory cortices, thereby boosting local/global processing. In contrast, parietal regions have been implied in suppressing distractors in global-to-local tasks (Mevorach et al., 2010). Selection of global or local elements entails enhancement of the relevant level but also suppression of the irrelevant, distracting, level. Significantly, parietal activation was observed in the N2 time window, in which higher amplitude for local targets where observed over left occipital sensors and higher amplitudes for global targets over right sensors. In line with previous neuroimaging studies (Mevorach et al., 2010), we hypothesize that parietal cortices suppress the irrelevant target level information leading to selection and detection of the target on the appropriate level. Previous studies further support the hypothesis that meditation enhances top-down control processes (Brefczynski-Lewis et al., 2007; Chan and Woollacott, 2007; Jha et al., 2007; van den Hurk et al., 2010a), and along those lines, even anatomical changes (increased cortical thickness) in frontal cortex have been observed as a result of meditation (Lazar et al., 2005). 
In laboratory settings a participant can learn to engage attention onto a target or to disengage attention from a distracter more efficiently (Chun, 2000; Kelley and Yantis, 2009). However, such learning is often highly specific to the stimulus parameters and the task that is trained. Contrary to this, during meditation the practitioner does not only direct attention to one specific stimulus type during one specific task but rather attends to many different stimuli of various types and domains that occur in different mental contexts (Slagter et al., 2011). This characteristic appears to be central for the general effect obtained in meditation. In fact, current proposals state that greater transfer across contexts is a function of the constant switching between processing priorities and stimulus material (Bherer et al., 2005). This may explain why the abilities that are trained during meditation are not specific to the training context but rather generalize across many different tasks and contexts. For instance, Slagter et al. (2007) found that by training the mechanisms of engagement and disengagement, meditators could more efficiently allocate attentional resources in time. By reducing the allocation of attentional resources to the first target item in an attentional blink paradigm, as reflected in a reduction in the $\mathrm{P} 3 \mathrm{~b}$, meditators had sufficient resources available to more effectively process a second target item (Slagter et al., 2007). Here, we show that developing these skills does not only alter attention in the temporal but also in the spatial domain. However, there is an important difference between our findings and those of Slagter et al. (2007). Namely, while they showed that decreasing the attentional resources devoted to T1 enhances performance, our data points to an earlier processing of information and an enhanced depth of processing. While previous reports and ours suggest that meditation exerts general effects on attention, it is yet unclear whether those benefits might come at a cost. Similar improvements in cognitive functions such as attention, visual discrimination, and decision making have been observed in video game playing, alongside with undesirable outcomes, such as an increased risk of addictive behavior (Bavelier et al., 2011). In contrast, meditation is proposed to foster emotional processing and well-being (Lutz et al., 2008a). While the latter points to an advantage of meditation over other forms of training, it is important to keep in mind that the limits and undesirable outcomes involved in meditation have not yet been extensively explored. Future research directly investigating those aspects will be extremely valuable. In addition, it remains a challenge to identity the specific aspects of the practice of meditation that train attention, as well as the underlying neural mechanism that govern those plastic changes. Identifying these aspects may allow for the development of mental

\section{REFERENCES}

Bavelier, D., Green, C. S., Han, D. H., Renshaw, P. F., Merzenich, M. M., and Gentile, D. A. (2011). Brains on video games. Nat. Rev. Neurosci. 12, 763-768.

Bherer, L., Kramer, A. F., Peterson, M. S., Colcombe, S., Erickson, K., and Becic, E. (2005). Training effects on dual-task performance: are there age-related differences in plasticity of attentional control? Psychol. Aging 20, 695-709.
Brefczynski-Lewis, J. A., Lutz, A., Schaefer, H. S., Levinson, D. B., and Davidson, R. J. (2007). Neural correlates of attentional expertise in long-term meditation practitioners. Proc. Natl. Acad. Sci. U.S.A. 104, 11483-11488.

Carter, O. L., Presti, D. E., Callistemon, C., Ungerer, Y., Liu, G. B., and Pettigrew, J. D. (2005). Meditation alters perceptual rivalry in Tibetan Buddhist monks. Curr. Biol. 15, R412-R413.

training programs which could be used in clinical and educational programs, allowing to bridge basic research with more applied context.

Although this study offers a unique opportunity to investigate brain plasticity in a homogenous population of monks and nuns, the current results must be interpreted with caution as sample sizes were small. In addition, it could be argued that these results merely reflect a bias in the selection of individuals with differing behavioral dispositions or even different lifestyles. This is very unlikely because the behavioral modulation found in the longitudinal study further demonstrates that meditation does affect spatial attention. Given this finding and evidence from other longitudinal studies that meditation is the causal factor in changes in attention (Slagter et al., 2007; Lutz et al., 2009), it seems most likely that the behavioral and electrophysiological differences found in our cross-sectional design represent changes in attentional skills that are trained during meditation and do not reflect a selection bias. Finally, we showed that individual differences in the global precedence effect covaried with the magnitude of the neural global vs. local effect, providing further support for the interpretation that our results are driven by changes in attention and not by changes in another factor such as motivation.

\section{SUMMARY}

Our results show that meditation can enhance performance on a hierarchical spatial attention task and that changes in spatial attention can already occur after a 4-day meditation intervention. Amplitude changes in the early P1 and N1 components indicate an earlier uptake of information in the meditators. Furthermore, the increased frequency at which target information was selected and the increased engagement of cortical networks previously related to the allocation of attention during global/local processing (Fink et al., 1997; Yamaguchi et al., 2000; Weissman et al., 2002), in the meditation population suggests both an increased depth of processing and an improved top-down allocation of attention in space.

\section{ACKNOWLEDGMENTS}

We would like to thank E. Rosales for help with EEG data acquisition, A. Mayer for help with the source analysis and C. M. Schwiedrzik for helpful comments on a previous version of the manuscript. We are indebted to the members of the EEG lab at the Max Planck Institute for Brain Research for helpful discussions and to the two reviewers for their insightful comments. This work was supported by the Max Planck Society.

Chan, D., and Woollacott, M. (2007). Effects of level of meditation experience on attentional focus: is the efficiency of executive or orientation networks improved? J. Altern. Complement. Med. 13, 651-657.

Chun, M. M. (2000). Contextual cueing of visual attention. Trends Cogn. Sci. 4, 170-178.

Chun, M. M., and Marois, R. (2002). The dark side of visual attention. Curr. Opin. Neurobiol. 12, 184-189.
Conci, M., Tollner, T., Leszczynski, M., and Muller, H. J. (2011). The time-course of global and local attentional guidance in Kanizsafigure detection. Neuropsychologia 49, 2456-2464.

Dalrymple, K. A., Kingstone, A., and Handy, T. C. (2009). Event-related potential evidence for a dual-locus model of global/local processing. Cogn. Neuropsychol. 26, 456-470.

Dehaene, S., Naccache, L., Cohen, L., Bihan, D. L., Mangin, J. F., Poline, 
J. B., and Riviere, D. (2001). Cerebral mechanisms of word masking and unconscious repetition priming. Nat. Neurosci. 4, 752-758.

Dehaene, S., Naccache, L., Le Clec'h, G., Koechlin, E., Mueller, M., DehaeneLambertz, G., Van de Moortele, P. F., and Le Bihan, D. (1998). Imaging unconscious semantic priming. Nature 395, 597-600.

Delorme, A., and Makeig, S. (2004). EEGLAB: an open source toolbox for analysis of single-trial EEG dynamics including independent component analysis. J. Neurosci. Methods 134, 9-21.

Dulaney, C. L., and Marks, W. (2007). The effects of training and transfer on global/local processing. Acta Psychol. 125, 203-220.

Evans, M. A., Shedden, J. M., Hevenor, S. J., and Hahn, M. C. (2000). The effect of variability of unattended information on global and local processing: evidence for lateralization at early stages of processing. Neuropsychologia 38, 225-239.

Fink, G. R., Halligan, P. W., Marshall, J. C., Frith, C. D., Frackowiak, R. S., and Dolan, R. J. (1997). Neural mechanisms involved in the processing of global and local aspects of hierarchically organized visual stimuli. Brain 120(Pt 10), 1779-1791.

Friston, K., Harrison, L., Daunizeau, J., Kiebel, S., Phillips, C., TrujilloBarreto, N., Henson, R., Flandin, G., and Mattout, J. (2008). Multiple sparse priors for the M/EEG inverse problem. Neuroimage 39, 1104-1120.

Green, C. S., and Bavelier, D. (2003). Action video game modifies visual selective attention. Nature 423, 534-537.

Green, C. S., and Bavelier, D. (2008). Exercising your brain: a review of human brain plasticity and traininginduced learning. Psychol. Aging 23, 692-701.

Han, S., and Chen, L. (1996). Processing of global and local propertiesan analysis with event-related brain potentials. Sci. China C Life Sci. 39, 179-188.

Han, S., He, X., and Woods, D. L. (2000). Hierarchical processing and level-repetition effect as indexed by early brain potentials. Psychophysiology 37, 817-830.

Han, S., He, X., Yund, E. W., and Woods, D. L. (2001). Attentional selection in the processing of hierarchical patterns: an ERP study. Biol. Psychol. 56, 113-130.

Hopf, J. M., Luck, S. J., Boelmans, K., Schoenfeld, M. A., Boehler, C. N., Rieger, J., and Heinze, H. J. (2006).
The neural site of attention matches the spatial scale of perception. $J$. Neurosci. 26, 3532-3540.

Jancke, L. (2009). The plastic human brain. Restor. Neurol. Neurosci. 27, 521-538.

Jha, A. P., Krompinger, J., and Baime, M. J. (2007). Mindfulness training modifies subsystems of attention. Cogn. Affect. Behav. Neurosci. 7 , 109-119.

Jiang, Y., and Han, S. (2005). Neural mechanisms of global/local processing of bilateral visual inputs: an ERP study. Clin. Neurophysiol. 116, 1444-1454.

Kastner, S., and Ungerleider, L. G. (2000). Mechanisms of visual attention in the human cortex. Annu. Rev. Neurosci. 23, 315-341.

Kelley, T. A., and Yantis, S. (2009) Learning to attend: effects of practice on information selection. J. Vis. $9,16$.

Lazar, S. W., Kerr, C. E., Wasserman, R. H., Gray, J. R., Greve, D. N., Treadway, M. T., Mcgarvey, M., Quinn, B. T., Dusek, J. A., Benson, H., Rauch, S. L., Moore, C. I., and Fischl, B. (2005). Meditation experience is associated with increased cortical thickness. Neuroreport 16, 1893-1897.

Lemmens, J. S., Valkenburg, P. M., and Peter, J. (2011). The effects of pathological gaming on aggressive behavior. J. Youth Adolesc. 40, 38-47.

Lin, Z., Lin, Y., and Han, S. (2008). Selfconstrual priming modulates visual activity underlying global/local perception. Biol. Psychol. 77, 93-97.

Lutz, A., Brefczynski-Lewis, J., Johnstone, T., and Davidson, R. J. (2008a). Regulation of the neural circuitry of emotion by compassion meditation: effects of meditative expertise. PLoS ONE 3, e1897. doi:10.1371/journal.pone.0001897

Lutz, A., Slagter, H. A., Dunne, J. D., and Davidson, R. J. (2008b). Attention regulation and monitoring in meditation. Trends Cogn. Sci. 12, 163-169.

Lutz, A., Slagter, H. A., Rawlings, N. B., Francis, A. D., Greischar, L. L., and Davidson, R. J. (2009). Mental training enhances attentional stability: neural and behavioral evidence. J. Neurosci. 29, 13418-13427.

Machinskaia, R. I., Krupskaia, E. V., and Kurganskaii, A. V. (2010). [Functional brain organization of global and local visual perception: an ERP study]. Fiziol. Cheloveka. 36, 29-48.

MacLean, K. A., Ferrer, E., Aichele, S. R., Bridwell, D. A., Zanesco, A. P., Jacobs, T. L., King, B. G., Rosenberg, E. L., Sahdra, B. K., Shaver, P. R., Wallace, B. A., Mangun, G. R., and Saron,
C. D. (2010). Intensive meditation training improves perceptual discrimination and sustained attention. Psychol. Sci. 21, 829-839.

Makovski, T., Vazquez, G. A., and Jiang, Y. V. (2008). Visual learning in multiple-object tracking. PLoS ONE 3, e2228. doi:10.1371/journal.pone. 0002228

Mevorach, C., Hodsoll, J., Allen, H., Shalev, L., and Humphreys, G. (2010). Ignoring the elephant in the room: a neural circuit to downregulate salience. J. Neurosci. 30 6072-6079.

Murray, M. M., Foxe, J. J., Higgins, B. A., Javitt, D. C., and Schroeder, C. E. (2001). Visuo-spatial neural response interactions in early cortical processing during a simple reaction time task: a high-density electrical mapping study. Neuropsychologia $39,828-844$.

Navon, D. (1977). Forest before trees: the precedence of global features in visual perception. Cogn. Psychol. 9, 353-383.

Proverbio, A. M., Minniti, A., and Zani, A. (1998). Electrophysiological evidence of a perceptual precedence of global vs. local visual information. Brain Res. Cogn. Brain Res. 6 321-334.

Rauscher, F. H., Shaw, G. L., and Ky, K. N. (1993). Music and spatial task performance. Nature 365, 611 .

Robertson, L. C., Lamb, M. R., and Knight, R. T. (1991). Normal globallocal analysis in patients with dorsolateral frontal lobe lesions. Neuropsychologia 29, 959-967.

Slagter, H. A., Davidson, R. J., and Lutz, A. (2011). Mental training as a tool in the neuroscientific study of brain and cognitive plasticity. Front. Hum. Neurosci. 5:17. doi:10.3389/fnhum.2011.00017

Slagter, H. A., Lutz, A., Greischar, L. L., Francis, A. D., Nieuwenhuis, S., Davis, J. M., and Davidson, R. J. (2007). Mental training affects distribution of limited brain resources. PLoS Biol. 5, e138. doi:10.1371/journal.pbio.0050138

Stoffer, T. H. (1994). Attentional zooming and the global-dominance phenomenon: effects of level-specific cueing and abrupt visual onset. Psychol. Res. 56, 83-98.

Thorpe, S., Fize, D., and Marlot, C. (1996). Speed of processing in the human visual system. Nature 381 , 520-522.

Valentine, E. R. S., and Philip, L. G. (1999). Meditation and attention: a comparison of the effects of concentrative and mindfulness. Ment. Health Relig. Cult. 2, 59-70. van den Hurk, P. A., Giommi, F., Gielen, S. C., Speckens, A. E., and Barendregt, H. P. (2010a). Greater efficiency in attentional processing related to mindfulness meditation. Q. J. Exp. Psychol. 63, 1168-1180.

van den Hurk, P. A., Janssen, B. H., Giommi, F., Barendregt, H. P., and Gielen, S. C. (2010b). Mindfulness meditation associated with alterations in bottom-up processing: psychophysiological evidence for reduced reactivity. Int. J. Psychophysiol. 78, 151-157.

van Leeuwen, S., Muller, N. G., and Melloni, L. (2009). Age effects on attentional blink performance in meditation. Conscious. Cogn. 18, 593-599.

Volberg, G., and Hubner, R. (2004). On the role of response conflicts and stimulus position for hemispheric differences in global/local processing: an ERP study. Neuropsychologia 42, 1805-1813.

Weinstein, A. M. (2010). Computer and video game addiction-a comparison between game users and non-game users. Am. J. Drug Alcohol Abuse 36, 268-276.

Weissman, D. H., Mangun, G. R., and Woldorff, M. G. (2002). A role for top-down attentional orienting during interference between global and local aspects of hierarchical stimuli. Neuroimage 17, 1266-1276.

Yamaguchi, S., Yamagata, S., and Kobayashi, S. (2000). Cerebral asymmetry of the "top-down" allocation of attention to global and local features. J. Neurosci. 20, RC72.

Conflict of Interest Statement: The authors declare that the research was conducted in the absence of any commercial or financial relationships that could be construed as a potential conflict of interest.

Received: 29 November 2011; accepted: 25 April 2012; published online: 15 May 2012.

Citation: van Leeuwen S, Singer $W$ and Melloni L (2012) Meditation increases the depth of information processing and improves the allocation of attention in space. Front. Hum. Neurosci. 6:133. doi: 10.3389/fnhum.2012.00133

Copyright ( 2012 van Leeuwen, Singer and Melloni. This is an open-access article distributed under the terms of the Creative Commons Attribution Non Commercial License, which permits noncommercial use, distribution, and reproduction in other forums, provided the original authors and source are credited. 


\section{APPENDIX}

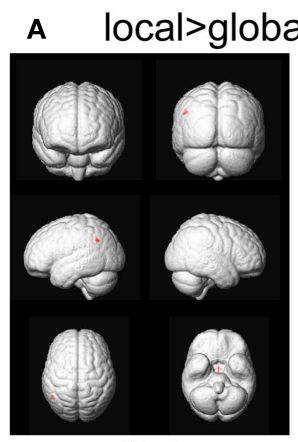

meditators

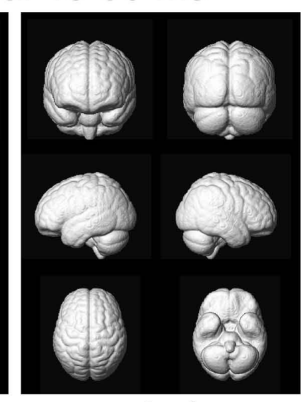

controls

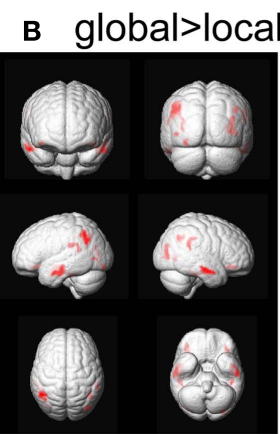

meditators

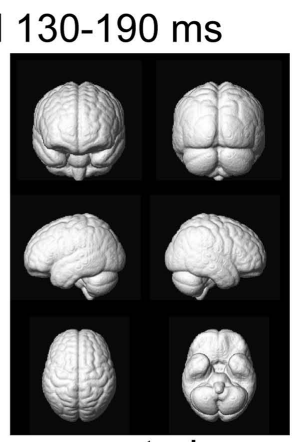

controls

c global vs. local $200-250 \mathrm{~ms}$

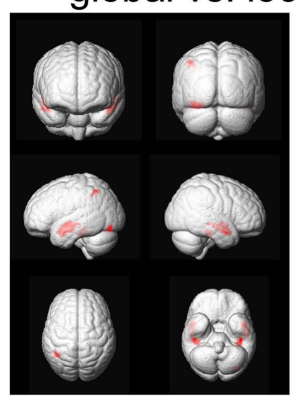

meditators

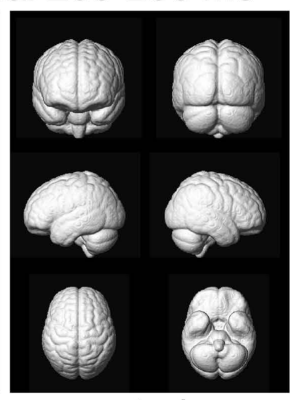

controls

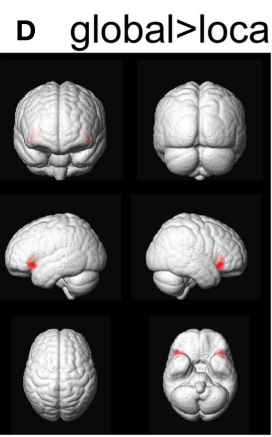

meditators

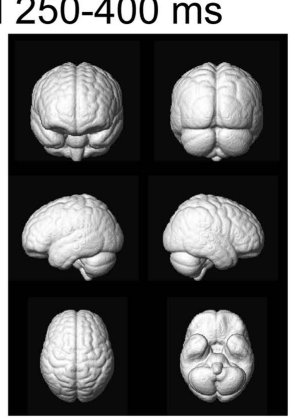

controls

FIGURE A1 | Source reconstruction. Areas showing a significant target level effect, $p<0.05$, FWE-corrected for meditators and controls separately. The analysis per group further validates the interaction effects observed in Figure 9, as the global vs. local effects are mostly found in the meditator group. 